"This paper is a preprint of a paper accepted by [IET Control Theory \& Applications, 2016, 10 (11)] and is subject to Institution of Engineering and Technology Copyright. When the final version is published, the copy of record will be available at IET Digital Library" 


\title{
Stabilizing the networked control systems involving actuation and measurement consecutive packet losses
}

\author{
Ahmadreza Argha ${ }^{1}, \mathrm{Li} \mathrm{Li}^{1}$, Steven $\mathrm{Su}^{1, *}$, Hung Nguyen ${ }^{1}$ \\ ${ }^{1}$ Faculty of Engineering and Information Technology, University of Technology, Sydney, PO Box \\ 123, Broadway, NSW 2007, Australia. \\ *Steven.Su@uts.edu.au
}

\begin{abstract}
This paper is devoted to the problem of designing a robust output-feedback discrete-time sliding mode control (ODSMC) for the networked systems involving both measuring and actuating data packet losses. Packet losses in the networked control systems (NCSs) have been modeled by utilizing the probability and the characteristics of the sources and the destinations. Here, the wellknown Bernoulli random binary distribution is used to model consecutive packet losses in the NCSs. In this paper, firstly, a robust observer-based discrete-time sliding mode control (DSMC) is proposed for the NCSs including random packet losses. The packet losses occur in the channels from the sensors to the controller and the channels from the controller to the actuators. Then, using the notion of exponential mean square stability, the boundedness of the obtained closedloop system is analyzed with an LMI approach. Our proposed robust ODSMC can be applied to unstable NCSs, and there is no need to stabilize the underlying system in advance. Illustrative examples are presented to show the effectiveness of the proposed approach.
\end{abstract}

\section{Introduction}

Feedback control systems which utilize real-time networks in their dynamic process are referred to as Networked Control Systems (NCSs). The NCSs have some great advantages compared to the conventional control systems such as smaller wiring system, reduced overall cost, and lower cost of system diagnosis and maintenance etc. However, exploiting the communication network in the feedback control loop makes the stability analysis and the controller design much more complex. This fact is basically due to the possible delays and data packet dropouts which exist in the communication network (arising from its limited bandwidth) [1]. Consequently, the problem of controlling the networked systems involving random delays and dropouts has been a subject of interest among researchers in the recent decades; e.g. see [1-5].

The network delays are mainly time-varying or random. Therefore, the control frameworks which have been proposed for deterministic time-delay systems are not beneficial for the NCSs [6]. In order to model the time-varying communication delays several different probabilistic methods have been considered so far, and as a consequence, some new notions of stability such as the mean-square stability or the exponential mean-square stability have been proposed. For instance, [7] models the random delays in NCSs as Markov chain and also assumes the closed-loop systems as jump systems. Furthermore, in [1] the random delays are modeled as the Bernoulli binary distributed white sequence which can take values of zero or one with a known probability. Note that due to the practicality and simplicity of the binary random delay, this model has received more 
attentions for random communication delays in NCSs $[6,8,9]$.

Although a large number of the early investigations on the discrete-time sliding mode control (DSMC) have attempted to make a discrete-time counterpart to the continuous-time reachability conditions [10-12], it is stated that for DSMC, eliminating the discontinuous control part from the controller can remove the chattering issue [13-15]. The obtained control law is mainly called linear control law. In other words, DSMC does not necessarily require a variable structure discontinuous control strategy (VSDCS) [13-15]. References [13,14] demonstrate that using the pure linear control law can ensure that the state trajectories stay within a boundary layer around the predetermined ideal sliding surface for systems involving bounded matched uncertainties. Moreover, referring to the results presented in $[13,14]$, the use of a switching function in the control law may not necessarily improve the performance. This fact would be the key feature of the observer-based output-feedback discrete-time SMC (ODSMC) proposed in this paper for NCSs involving random measuring and actuating delays.

Most of the work on SMC has been implemented for the systems in which system signals are transmitted without any packet losses. Recently, designing a sliding mode controller subject to packet losses has been carried out in $[16,17]$. The discrete-time sliding mode controllers given in the existing literature are derived based on several assumptions such as:

1) the packet losses occur only in the channel from the sensor to the controller;

2) the system states are entirely available;

3) the packet dropouts may not occur successively.

These are clearly unrealistic assumptions for many practical problems. This work intends to design sliding mode controllers for the NCSs involving consecutive packet losses (or long-term random delays) in both measurement and actuation channels, using only output information. This ODSMC can distinguish itself from the existing literature on the SMCs applied to the NCSs, in the sense that both the measurement and actuation delays are viewed as the Bernoulli distributed white sequence. This matter has been considered for the design of other control strategies such as observer-based $H_{\infty}$ and state-feedback controllers [1,4].

In brief, the main goal of this paper is to stabilize an NCS involving measurement and actuation packet dropouts with an observer-based ODSMC. The main contribution of this paper includes the following major innovations.

- This note revises the observer utilized in $[1,4]$ to a more practical alternative. Indeed, $[1,4]$ assume that the state observer and controller are not located in the same place, or equivalently, the control signals used in the state observer involve the random time-delays that exist in the channel from the system controller to the actuators. This could not be a real assumption in most of the NCSs.

- The proposed ODSMC can be applied to the NCSs involving both the measuring and actuating consecutive packet losses.

- The proposed robust ODSMC scheme provides an integrated framework for general systems. This is certainly different from the DSMC introduced in [16] which can only be applied to the stable systems, and [18] which stabilizes the underlying system first and then designs the sliding mode controller separately.

- In order to derive the controller or analyze the boundedness of the underlying closed-loop system, the system state is not assumed to be bounded in this paper; see [16, 19, 20]. The system uncertainty will be addressed using the robust control techniques. 
- A novel method is developed to reduce the conservatism existing in the current literature that removes the cross term between the system state and disturbance to make a fully diagonal problem.

- The proposed control law in this paper does not belong to the class of high-gain control laws, unlike other cited references for DSMC and ODSMC.

The rest of this paper is organized as follows: Section 2 describes the problem formulation. Section 3 presents the proposed approach to designing the sliding surface and ODSMC. Effectiveness of the proposed ODSMC is studied by numerical examples in Section 4. Finally, Section 5 concludes this paper.

Notation: $\left[\Sigma_{i j}\right]_{r \times s}$ is a block matrix with the block entries $\Sigma_{i j}, i=1, \cdots, r, j=1, \cdots, s$.

\section{Problem Formulation and Preliminaries}

\subsection{Problem statement}

Consider the NCS with (consecutive) random packet losses as shown in Fig. 1. As seen the NCS

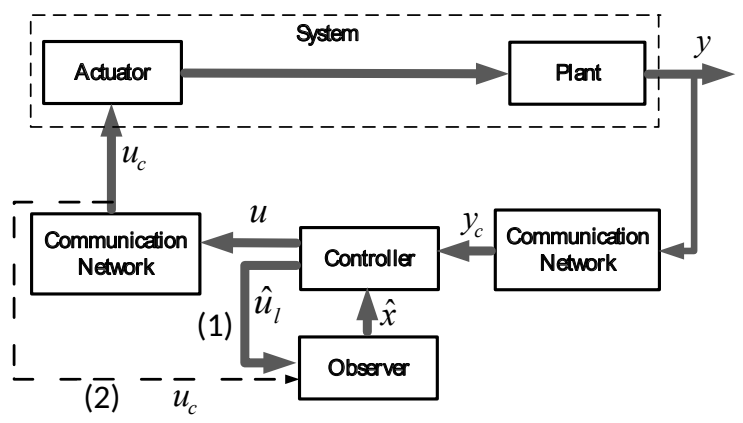

Fig. 1. NCS structure: (1) control signal to the observer in the proposed approach, (2) control signal to the observer in the approach in $[1,4]$

here involves both the measurement and actuation packet dropouts. In other words, the system does not have the access to the control input $u(k)$, generated by the controller, and instead it would utilize the communicated control input $u_{c}(k)$. On the other hand, due to the packet losses existing in the channel from the sensors to the controller, the observer which is at the same location of the controller also does not receive the actual values of the system output $y(k)$, but $y_{c}(k)$. The observer will provide the state estimate $\hat{x}(k)$ for the controller using the signal $\hat{u}_{l}(k)$, which will be defined later in this paper, rather than $u(k)(c f .[1,4])$.

Now, consider the following uncertain linear discrete-time system which explains the dynamics in Fig. 1,

$$
\text { System : }\left\{\begin{array}{l}
x(k+1)=[A+\Delta A(k)] x(k)+B\left[u_{c}(k)+d(k)\right] \\
y(k)=C x(k),
\end{array}\right.
$$

where $x(k) \in \mathbb{R}^{n}, u_{c}(k) \in \mathbb{R}^{m}$ and $y(k) \in \mathbb{R}^{p}$ are the system state, communicated control input (see Fig. 1) and system output respectively. Without loss of generality, it is assumed that $m \leq n$, $\operatorname{rank}(B)=m$ and $\operatorname{rank}(C)=p$. Besides, it is assumed that $(A, B)$ is controllable and $(A, C)$ is observable. The uncertain matrix $\Delta A(k)$ has the form of

$$
\Delta A(k)=M R(k) N,
$$


where $R(k) \in \mathbb{R}^{q \times q}$ is an unknown matrix satisfying $R^{T}(k) R(k) \leq I, \forall k \geq 0$ and matrices $M \in \mathbb{R}^{n \times q}$ and $N \in \mathbb{R}^{q \times n}$ are known; $d(k)$ denotes the external disturbance with known bound, $\|d(k)\| \leq \bar{d}$, where $\bar{d}>0$.

As the measured outputs are sent to the controller via a communication network, the measurements may involve randomly varying communication delays and/or the detrimental phenomena referred to as data packet dropouts. The communicated output $y_{c}(k)$ and communicated input $u_{c}(k)$ are assumed to be as

$$
\begin{aligned}
& \text { Communicated output : }\left\{\begin{array}{l}
x_{c}(k)=(1-\alpha(k)) x(k)+\alpha(k) x_{c}(k-1) \\
y_{c}(k)=C x_{c}(k),
\end{array}\right. \\
& \text { Communicated input : } u_{c}(k)=(1-\beta(k)) u(k),
\end{aligned}
$$

where $x_{c}(k)$ denotes the communicated system state which is not available and the stochastic variables $\alpha(k) \in \mathbb{R}$ and $\beta(k) \in \mathbb{R}$ are Bernoulli distributed white sequences with

$$
\begin{aligned}
& \left\{\begin{array}{l}
\operatorname{Prob}\{\alpha(k)=1\}=\mathbb{E}\{\alpha(k)\}=\bar{\alpha} \\
\operatorname{Prob}\{\alpha(k)=0\}=1-\mathbb{E}\{\alpha(k)\}=1-\bar{\alpha},
\end{array}\right. \\
& \left\{\begin{array}{l}
\operatorname{Prob}\{\beta(k)=1\}=\mathbb{E}\{\beta(k)\}=\bar{\beta} \\
\operatorname{Prob}\{\beta(k)=0\}=1-\mathbb{E}\{\beta(k)\}=1-\bar{\beta},
\end{array}\right.
\end{aligned}
$$

$0 \leq \bar{\alpha}<1,0 \leq \bar{\beta}<1$ imply the probability of a data packet loss. The stochastic variable $\beta(k)$ is independent of the stochastic variable $\alpha(k)$. Notice that $u_{c}(k)$ involves a random communication delay similar to that in the measurement communication, however, with a different switching probability. It can be frequently seen in the literature to use a similar Bernoulli distributed white sequence model but with different switching probability for the random delays in the signals from the sensors to the controller and also in the signals from the controller to the actuators; see e.g. $[1,4]$. Besides, two different schemes for the systems involving packet loss have been considered, i.e., the hold-input method (which is used here in the communicated output $y_{c}(k)$ in (3)) and the zero-input method (which is used here in the communicated input $u_{c}(k)$ in (4)) [21-23]. The holdinput method seems to have better performance than the zero-input one. However, according to the existing literature (e.g. see [21, 23]), either of them cannot outperform the other, even in simple scalar systems [23]. Therefore, here, for simplification purpose, the zero-input method would be utilized to deal with the packet dropouts in the channel from the controller to the actuators.

In this note, we use the following estimation scheme to provide the controller with the system state information,

$$
\text { Observer : }\left\{\begin{array}{l}
\hat{x}(k+1)=A \hat{x}(k)+B \hat{u}_{l}(k)+\frac{1}{1-\bar{\alpha}} L\left[y_{c}(k)-\hat{y}_{c}(k)\right] \\
\hat{u}_{l}(k)=(1-\bar{\beta}) u_{l}(k) \\
\hat{y}_{c}(k)=(1-\bar{\alpha}) C \hat{x}(k)+\bar{\alpha} C \hat{x}(k-1),
\end{array}\right.
$$

where $\hat{x}(k) \in \mathbb{R}^{n}$ is the state estimate of the system in (1), $\hat{y}_{c}(k) \in \mathbb{R}^{p}$ is the observer output, $L \in \mathbb{R}^{n \times p}$ is the observer gain and $u_{l}(k) \in \mathbb{R}^{m}$ denotes the linear part of the system controller $u(k)$ to be proposed in the following of this paper.

Remark 1. The measurement model (3) is fundamentally different from the one that has been used in $[1,4,6,9]$ etc. Note that in the literature, rather than the measurement model (3), the following one is frequently utilized,

$$
y_{c}(k)=(1-\alpha(k)) y(k)+\alpha(k) y(k-1) .
$$


This model indeed would impose an assumption on the system networks that the packet dropout may not occur successively. This is obviously not a realistic assumption. Instead, the model (3) can cope with longer random delays and/or frequent packet losses. Notice that the model (8) has been used to cope with both time varying communication delay (e.g. see $[1,4])$ and data packet dropout (e.g. see [16]). Considering the model (3) instead of model (8), if a packet loss occurs, the controller is provided with $y_{c}(k)=y_{c}(k-1)$, or the controller utilizes $y_{c}(k)=y(k)$ otherwise. Here, $y_{c}(k-1)$ denotes the last available communicated data packet. This justification can also be used for the communication random time-delay accordingly. It means that if the time-delay of the communication channel, which is assumed to be $\tau_{d}$, is less than a sampling period $(T)$ of the discrete-time control system, the delay has no influence on the system and we have $y_{c}(k)=y(k)$. But, if $\tau_{d} \geq T$, then $y_{c}(k)=y_{c}(k-1)$. Note that, in the case of random delay occurrence, $y_{c}(k-1)$ can be regarded as $y\left(k-\tau_{d}\right)$.

Notice also that we do not bound the number of possible consecutive packet losses (or equivalently the random time-delays $c f$. [4]). Indeed, there is an implicit stochastic constraint indicated by the Bernoulli variable $\alpha(k)$ as $\operatorname{Prob}\{\alpha(k)=1\}=\mathbb{E}\{\alpha(k)\}=\bar{\alpha}$. In simpler terms, if $\bar{\alpha}$ is a large value, the number of possible consecutive packet losses increases and vice versa.

Remark 2. Notice that in $[1,4]$, the observer in (7) is assumed to be as

$$
\hat{x}(k+1)=A \hat{x}(k)+B u_{l_{c}}(k)+L\left[y_{c}(k)-\hat{y}_{c}(k)\right],
$$

where $u_{l_{c}}$ denotes the linear part of $u_{c}(k)$. In other words, the control input utilized in the observer is the (linear part of the) communicated control input to the system which involves random communication delay. This itself means that either the observer and controller are not located in the same place together, or the observer has access to $u_{c}(k)$; see the dash-dotted line in Fig 1 . This structure does not seem to be a practical case in most of the NCSs. Therefore, to address this problem, in this paper, the control input $\hat{u}_{l}(k)$ is exploited in the observer (see (7)), which is more applicable for practical NCSs.

This paper will consider the problem of designing a robust observer-based DSMC for the NCS in (1)-(4). In the sequel of this paper, for simplification, the brief $\alpha, \beta$ and $\Delta A$ are used instead of $\alpha(k), \beta(k)$ and $\Delta A(k)$, respectively.

\subsection{Preliminaries}

The following lemmas and definition are useful in the sequel of this paper.

Lemma 1 ([24]). Let $E, F(k)$ and $G$ be real matrices of appropriate dimensions with $F^{T}(k) F(k) \leq$ $I, \forall k \geq 0$. Then, for any scalar $\varepsilon>0$, we have

$$
E F(k) G+G^{T} F^{T}(k) E^{T} \leq \varepsilon E E^{T}+\varepsilon^{-1} G^{T} G .
$$

Lemma 2. Let $\tilde{E}, \tilde{H}$ and $\Xi$ be real matrices of appropriate dimensions. Then, for any matrix $\Xi>0$, we have

$$
\tilde{E}^{T} \tilde{H}+\tilde{H}^{T} \tilde{E} \leq \tilde{E}^{T} \Xi \tilde{E}+\tilde{H}^{T} \Xi^{-1} \tilde{H} .
$$

Proof. It can easily be proved by

$$
\left[\tilde{E}^{T}-\tilde{H}^{T}\left(\Xi^{T}\right)^{-1}\right] \Xi\left[\tilde{E}-\Xi^{-1} \tilde{H}\right] \geq 0 .
$$


Lemma 3. Consider the following inequality,

$$
\Gamma\left(X_{1}, X_{2}, \cdots, X_{n}\right)-\sum_{i=1}^{n} \digamma_{i}^{T}\left(X_{i}\right) \Lambda_{i}^{-1}\left(X_{i}\right) \digamma_{i}\left(X_{i}\right)<0,
$$

where $X_{i}, i=1, \cdots, n$ are the matrix variables, $\Gamma(\cdot)$ is symmetric, $\Lambda_{i}\left(X_{i}\right)>0$ and $\digamma_{i}\left(X_{i}\right)$ are functions of $X_{i}, i=1, \cdots, n$. Then the inequality in (9) is feasible in $X_{i}, i=1, \cdots, n$ if and only if there exist matrices $J_{i}, i=1, \cdots, n$ such that the following inequality is feasible in $X_{i}, J_{i}, i=1, \cdots, n$,

$$
\Gamma\left(X_{1}, X_{2}, \cdots, X_{n}\right)+\sum_{i=1}^{n}\left(J_{i}^{T} \Lambda_{i}\left(X_{i}\right) J_{i}+J_{i}^{T} \digamma_{i}\left(X_{i}\right)+\digamma_{i}^{T}\left(X_{i}\right) J_{i}\right)<0 .
$$

Proof. It can be shown that the feasibility of (9) is equivalent to the feasibility of

$$
\begin{aligned}
& \Gamma\left(X_{1}, X_{2}, \cdots, X_{n}\right)-\sum_{i=1}^{n} \digamma_{i}^{T}\left(X_{i}\right) \Lambda_{i}^{-1}\left(X_{i}\right) \digamma_{i}\left(X_{i}\right) \\
& +\sum_{i=1}^{n}\left(\left[J_{i}+\Lambda_{i}^{-1}\left(X_{i}\right) \digamma_{i}\left(X_{i}\right)\right]^{T} \Lambda_{i}\left(X_{i}\right)\left[J_{i}+\Lambda_{i}^{-1}\left(X_{i}\right) \digamma_{i}\left(X_{i}\right)\right]\right)<0,
\end{aligned}
$$

where $J_{i}, i=1, \cdots, n$ are introduced auxiliary variables [25]. Then, it is easy to show that the above inequality is the same as (10). This completes the proof.

Also according to [1] the notion of exponential mean square stability for the stochastic parameter system is as follows.

Definition 1 ([1]). Consider the following stochastic system,

$$
\xi(k+1)=\bar{A} \xi(k),
$$

where $\xi(k) \in \mathbb{R}^{n}$ denotes the state vector of the stochastic system and $\xi(0) \in \mathbb{R}^{n}$ is its initial condition. If there exist constants $\gamma>0$ and $\rho \in(0,1)$ such that

$$
\mathbb{E}\left\{\|\xi(k)\|^{2}\right\} \leq \gamma \rho^{k} \mathbb{E}\left\{\|\xi(0)\|^{2}\right\}, \quad \forall k>0,
$$

then the stochastic system in (11) is said to be exponentially mean square stable.

Lemma 4 ([1]). Let $V(\xi(k))$ be a Lyapunov function. If there exist real scalars $\kappa \geq 0, \mu>0$, $v>0$ and $0<\psi<1$ such that

$$
\mu\|\xi(k)\|^{2} \leq V(\xi(k)) \leq v\|\xi(k)\|^{2},
$$

and

$$
\mathbb{E}\{V(\xi(k+1)) \mid \xi(k)\}-V(\xi(k)) \leq \kappa-\psi V(\xi(k)),
$$

then the sequence $\xi(k)$ satisfies

$$
\mathbb{E}\left\{\|\xi(k)\|^{2}\right\} \leq \frac{v}{\mu} \mathbb{E}\left\{\|\xi(0)\|^{2}\right\}(1-\psi)^{k}+\frac{\kappa}{\mu \psi} .
$$

Moreover, the term $\frac{\kappa}{\mu \psi}$ will be called the ultimate bound of $\mathbb{E}\left\{\|\xi(k)\|^{2}\right\}$. 
Lemma 4 introduces a special boundedness definition for stochastic discrete-time systems involving exogenous disturbances. In this paper, a system state which satisfies the condition presented in Lemma 4 is said to be exponentially mean square bounded.

Lemma 5 ([26]). For a given $B \in \mathbb{R}^{n \times m}$ with $\operatorname{rank}(B)=m$, and

$$
B=U\left[\begin{array}{l}
\Sigma \\
0
\end{array}\right] V^{T},
$$

where $U \in \mathbb{R}^{n \times n}$ and $V \in \mathbb{R}^{m \times m}$ are two orthogonal matrices and $\Sigma:=\operatorname{diag}\left(\sigma_{1}, \cdots, \sigma_{m}\right), \sigma_{i},(i=$ $1, \cdots, m)$ denote nonzero singular values of $B$, given that $0<P \in \mathbb{R}^{n \times n}$, then there exists $Z \in \mathbb{R}^{m \times m}$ such that

$$
P B=B Z,
$$

if and only if $P$ has the following structure

$$
P=U^{T}\left[\begin{array}{cc}
P_{11} & 0 \\
0 & P_{22}
\end{array}\right] U
$$

where $0<P_{11} \in \mathbb{R}^{m \times m}, 0<P_{22} \in \mathbb{R}^{(n-m) \times(n-m)}$.

\section{Stochastic sliding mode control}

This section aims to design a robust observer-based ODSMC in order to stabilize the NCS in (1).

\subsection{Designing the sliding function subject to consecutive packet losses}

Consider the following discrete-time linear sliding function,

$$
\sigma(k)=S \hat{x}(k),
$$

where the matrix $S \in \mathbb{R}^{m \times n}$ will be designed later such that $S B$ is nonsingular. Note that in the ideal sliding mode we have

$$
\sigma(k)=0, \quad \forall k \geq k_{s}
$$

where $k_{s}>0$ denotes the time when sliding motion starts. Assume that $d(k)=\left[d_{1}(k), \cdots, d_{m}(k)\right]^{T}$, and

$$
d_{i}^{l} \leq d_{i}(k) \leq d_{i}^{u}, \quad i=1, \cdots, m
$$

Also, define,

$$
d_{i}^{a}=\frac{d_{i}^{u}+d_{i}^{l}}{2}, d_{i}^{b}=\frac{d_{i}^{u}-d_{i}^{l}}{2}, i=1, \cdots, m,
$$

and

$$
\mathfrak{D}^{a}=\left[d_{1}^{a}, \cdots, d_{m}^{a}\right]^{T}, \mathfrak{D}^{b}=\left[d_{1}^{b}, \cdots, d_{m}^{b}\right]^{T} .
$$

The controller is assumed to have the following structure,

$$
u(k)=-\frac{1}{1-\bar{\beta}}\left[(S B)^{-1}(S A-\Phi S) \hat{x}(k)+\vartheta(k)\right],
$$

where $\Phi \in \mathbb{R}^{m \times m}$ is a known stable matrix and $\vartheta(k)$ denotes the approximation of the disturbance $d(k)$ used in the controller to compensate the harmful effect of the disturbance. 
Remark 3. With a quick glimpse into the literature, it can be found that a frequently used candidate for the component $\vartheta(k)$ has the general form of:

$$
\vartheta(k)=\varphi+\varsigma \operatorname{sgn}(\sigma(k))
$$

where $\varphi$ and $\zeta$ are known parameters. Normally, $\varphi$ is simply selected as $\mathfrak{D}^{a}$. Notice that providing the discrete-time sliding mode controller with a switching component cannot necessarily improve the control performance and even can be detrimental to the control performance [13, 27]. However, some papers in the literature have claimed a better performance by using the discontinuous components. Indeed, these papers assume that either the sampling rate of the system is very high compared with the maximum frequency of the exogenous disturbance or the exogenous disturbance is slow (smooth and bounded). With either of these assumptions, the closed-loop system would behave more or less as a continuous-time system [15] and hence, using a discontinuous component in the controller may improve the performance. However, broadly speaking, the large switching gains in (19) may result in excessive actions in the actuators, and hence, it is worth mentioning that the switching gain must be chosen small.

It is also assumed that

$$
\|\vartheta(k)\| \leq \kappa\left\|\mathfrak{D}^{b}\right\|
$$

where $\kappa>0$ is a scalar and

$$
\left\|d_{\vartheta}(k)\right\| \leq \tau\left\|\mathfrak{D}^{b}\right\|
$$

where $d_{\vartheta}(k)=d(k)-\vartheta(k)$ and $\tau>0$ is a scalar. The term $(S B)^{-1} \Phi S \hat{x}(k)$, in $u(k)$ (see (18)), is used to govern the rate of convergence to the sliding manifold.

Remark 4. Notice that the literature usually assumes the bounds on $S \Delta A x(k)$ before the closedloop stability is proved; $c f$. $[16,19,20]$. It should be mentioned that since $\Delta A(k)$ is a time-varying uncertainty, in order to find the bounds of $S \Delta A x(k)$, one requires to know the behaviour of the closed-loop system state in advance, and as a result, to realize $\|x(k)\|$ from the beginning before applying the controller to the system. Broadly speaking, it is not a realistic assumption that we can have the bounds on the system states. Instead, our paper deals with the unmatched (mismatched) uncertainties $\triangle A x(k)$ by only using robust control strategies without assuming any a priori bound on the system states.

Here, similar to [28], it is assumed that $\Phi=\lambda I_{m}$, where $0 \leq \lambda<1$ is a given constant value. Thanks to the special form of $\Phi$ which can commute with $S$, the control law $u(k)$ in (18) can be written as

$$
\text { Controller : } \begin{aligned}
u(k) & =-\frac{1}{1-\bar{\beta}}\left[(S B)^{-1} S A_{\lambda} \hat{x}(k)+\vartheta(k)\right] \\
& \triangleq u_{l}(k)+u_{n}(k)
\end{aligned}
$$

where $A_{\lambda}=A-\lambda I_{n}$ and $u_{l}(k)$ is the linear part of $u(k)$ which was used in the observer design (7). Define the estimation errors as

$$
\text { Estimation error : }\left\{\begin{array}{l}
e(k):=x(k)-\hat{x}(k) \\
e_{c}(k):=x_{c}(k)-\hat{x}(k) .
\end{array}\right.
$$


Then, by applying the controller (22) to (1) and also using (23), (3) and (7), the closed-loop system is obtained as

\section{Closed-loop system :}

$$
\left\{\begin{aligned}
& x(k+1)= {\left[A+\Delta A-\hat{A}+\frac{\beta-\bar{\beta}}{1-\bar{\beta}} \hat{A}\right] x(k)+\left[1-\frac{\beta-\bar{\beta}}{1-\bar{\beta}}\right] \hat{A} e(k)+B\left[d_{\vartheta}(k)+\frac{\beta-\bar{\beta}}{1-\bar{\beta}} \vartheta(k)\right] } \\
& e(k+1)= {\left[\Delta A+\frac{\beta-\bar{\beta}}{1-\bar{\beta}} \hat{A}+\frac{\alpha-\bar{\alpha}}{1-\bar{\alpha}} L C\right] x(k)+\left[A-L C-\frac{\beta-\bar{\beta}}{1-\bar{\beta}} \hat{A}\right] e(k) } \\
& \quad-\frac{\alpha-\bar{\alpha}}{1-\bar{\alpha}} L C x_{c}(k-1)-\theta L C e_{c}(k-1)+B\left[d_{\vartheta}(k)+\frac{\beta-\bar{\beta}}{1-\bar{\beta}} \vartheta(k)\right] \\
& x_{c}(k)=(1-\alpha) x(k)+\alpha x_{c}(k-1) \\
& e_{c}(k)=-\alpha x(k)+\alpha x_{c}(k-1)+e(k)
\end{aligned}\right.
$$

where $\hat{A}=B(S B)^{-1} S A_{\lambda}$ and $\theta=\frac{\bar{\alpha}}{1-\bar{\alpha}}$. Also, it can simply be found that

$$
\sigma(k+1)=S\left(\lambda I-\frac{\alpha-\bar{\alpha}}{1-\bar{\alpha}} L C\right) x(k)-S(\lambda I-L C) e(k)+\frac{\alpha-\bar{\alpha}}{1-\bar{\alpha}} S L C x_{c}(k-1)+\theta S L C e_{c}(k-1) .
$$

\subsection{Stability analysis}

Notice that in the case of applying DSMC to discrete-time systems involving exogenous disturbances, the closed-loop system should be analyzed in terms of boundedness. Also, the proposed DSMC could only ensure the state trajectories to be driven into a boundary layer around the ideal sliding surface $\sigma(k)=0$. This issue is indeed regarded as the quasi sliding mode (QSM) in the literature. The following theorem considers a method to analyze simultaneously the reachabiltiy of QSM and the stability of the system states utilizing a discrete-time Lyapunov stability method, in the absence of exogenous disturbances. The characterization of the bounds on the closed-loop system states and sliding function is presented separately later in Theorem 2 . Further, as Theorem 2 needs to derive the cross terms between the system state (sliding function) and the components $d_{\vartheta}(k) / \vartheta(k)$, in order to avoid unnecessary repetition of the technical manipulations, we will start the proof of Theorem 1 more generally (with the external disturbance and the discontinuous component $\vartheta(k)$ ) for the sake of Theorem 2. We then let $\left[\begin{array}{c}d_{\vartheta}(k) \\ \vartheta(k)\end{array}\right]=0$ to derive the LMI condition for the stability analysis and controller/observer synthesis.

Theorem 1. In the absence of the exogenous disturbance $d(k)$, the control law (18) can steer the state of the stochastic system (1) onto the ideal sliding surface (14) and, also the system is exponentially mean square stable if there exist matrices $0<P:=U^{T}\left[\begin{array}{cc}P_{11} & 0 \\ 0 & P_{22}\end{array}\right] U, Q_{1}>0, Q_{2}>0$,

\begin{tabular}{|c|c|c|c|c|c|c|c|c|c|c|c|c|c|}
\hline$\check{\Sigma}_{11}$ & $\star$ & $\star$ & $\star$ & 夫 & $\star$ & $\star$ & $\star$ & $\star$ & $\star$ & $\star$ & 夫 & $\star$ & \\
\hline$\tilde{\Sigma}_{12}^{T}$ & $\tilde{\Sigma}_{22}$ & $\star$ & $\star$ & $\star$ & $\star$ & 夫 & $\star$ & $\star$ & $\star$ & $\star$ & $\star$ & $\star$ & \\
\hline$B X_{2}$ & $B X_{3}$ & $-P$ & $\star$ & $\star$ & $\star$ & $\star$ & $\star$ & $\star$ & $\star$ & $\star$ & $\star$ & $\star$ & \\
\hline$-\bar{\alpha} Q_{2}$ & $\bar{\alpha} Q_{2}$ & 0 & $\bar{\Sigma}_{33}$ & $\star$ & $\star$ & $\star$ & $\star$ & $\star$ & $\star$ & $\star$ & $\star$ & $\star$ & \\
\hline 0 & 0 & 0 & 0 & $-Q_{2}+\rho I$ & $\star$ & 夫 & $\star$ & $\star$ & $\star$ & $\star$ & $\star$ & $\star$ & \\
\hline$\lambda B^{T} P$ & $B^{T} P A_{\lambda}$ & 0 & 0 & 0 & $-B^{T} P B$ & 夫 & $\star$ & $\star$ & $\star$ & $\star$ & 夫 & $\star$ & \\
\hline$-\lambda B^{T} P$ & $B^{T}\left(\lambda P-X_{4} C\right)$ & 0 & 0 & $-\theta B^{T} X_{4} C$ & 0 & $-B^{T} P B$ & $\star$ & $\star$ & $\star$ & $\star$ & $\star$ & $\star$ & $<0$ \\
\hline$-2 \phi B^{T} P A_{\lambda}$ & $2 \phi B^{T} P A_{\lambda}$ & 0 & 0 & 0 & 0 & 0 & $-2 \phi B^{T} P B$ & $\star$ & $\star$ & $\star$ & $\star$ & $\star$ & \\
\hline 0 & 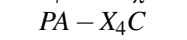 & 0 & 0 & $-\theta X_{4} C$ & 0 & 0 & 0 & $-P$ & $\star$ & $\star$ & $\star$ & $\star$ & \\
\hline$\theta X_{4} C$ & 0 & 0 & $-\theta X_{4} C$ & 0 & 0 & 0 & 0 & 0 & $-\theta P$ & $\star$ & $\star$ & $\star$ & \\
\hline$\theta B^{T} X_{4} C$ & 0 & 0 & $-\theta B^{T} X_{4} C$ & 0 & 0 & 0 & 0 & 0 & 0 & $-\theta B^{T} P B$ & 夫 & 夫 & \\
\hline$P A+B X_{1}$ & 0 & 0 & 0 & 0 & 0 & 0 & 0 & 0 & 0 & 0 & $-P$ & $\star$ & \\
\hline 0 & 0 & 0 & 0 & 0 & $M^{T} P B$ & 0 & 0 & $M^{T} P$ & 0 & 0 & $M^{T} P$ & $\begin{array}{c}-\varepsilon I \\
(25)\end{array}$ & \\
\hline
\end{tabular}
$X_{1}, X_{2}, X_{3}$ and $X_{4}$, and scalars $\varepsilon>0$ and $\rho>0$ satisfying the following LMI: 
where $M$ and $N$ are known matrices of the uncertainty in (2), $0<P_{22} \in \mathbb{R}^{(n-m) \times(n-m)}$ and $U \in$ $\mathbb{R}^{n \times n}$ is defined in Lemma $5, \check{\Sigma}_{11}=-P+(1-\bar{\alpha}) Q_{1}+\bar{\alpha} Q_{2}+X_{2}^{T} B^{T}+B X_{2}+\rho I+\varepsilon N^{T} N, \tilde{\Sigma}_{12}=$ $-X_{2}^{T} B^{T}+B X_{3}-\bar{\alpha} Q_{2}, \tilde{\Sigma}_{22}=-X_{3}^{T} B^{T}-B X_{3}-P+Q_{2}+\rho I, \bar{\Sigma}_{33}=-(1-\bar{\alpha}) Q_{1}+\bar{\alpha} Q_{2}+\rho I, \theta=$ $\frac{\bar{\alpha}}{1-\bar{\alpha}}, \phi=\frac{\bar{\beta}}{1-\bar{\beta}}$ and $\{\star\}$ denotes the symmetric elements in a symmetric matrix. Here $S=B^{T} P$ and the observer gain is

$$
L=P^{-1} X_{4}
$$

Proof. Refer to Appendix.

Remark 5. Note that the inequality (43) in the Appendix, which contains the negative quadratic signum terms, cannot easily be converted to an LMI. Let us explain the technique that we utilized in the proof of Theorem 1 to deal with one of the negative terms. Obviously, $-\sigma^{T}(k)(S B)^{-1} \sigma(k)$ in $\Delta V(\zeta(k))$ can be rewritten as

$$
\begin{aligned}
& -\sigma^{T}(k)(S B)^{-1} \sigma(k) \\
= & -\left[x^{T}(k) e^{T}(k)\right]\left[\begin{array}{c}
I \\
-I
\end{array}\right] S^{T}(S B)^{-1} S\left[\begin{array}{c}
I \\
-I
\end{array}\right]^{T}\left[\begin{array}{l}
x(k) \\
e(k)
\end{array}\right] .
\end{aligned}
$$

Hence, according to Lemma 3, the feasibility of

$$
\tilde{\Psi}-\left[\begin{array}{c}
I \\
-I \\
0 \\
\vdots \\
0
\end{array}\right] S^{T}(S B)^{-1} S\left[\begin{array}{c}
I \\
-I \\
0 \\
\vdots \\
0
\end{array}\right]<0
$$

where

$$
\tilde{\Psi}=\Psi+\left[\begin{array}{c}
I \\
-I \\
0 \\
\vdots \\
0
\end{array}\right] S^{T}(S B)^{-1} S\left[\begin{array}{c}
I \\
-I \\
0 \\
\vdots \\
0
\end{array}\right]^{T}
$$

and $\Psi$ is the left hand side of the inequality in (43), is equivalent to that of

$$
\tilde{\Psi}+\left[\begin{array}{c}
F_{2}^{T} \\
F_{3}^{T} \\
0 \\
\vdots \\
0
\end{array}\right](S B)\left[\begin{array}{c}
F_{2}^{T} \\
F_{3}^{T} \\
0 \\
\vdots \\
0
\end{array}\right]^{T}+\left[\begin{array}{c}
F_{2}^{T} \\
F_{3}^{T} \\
0 \\
\vdots \\
0
\end{array}\right] S\left[\begin{array}{c}
I \\
-I \\
0 \\
\vdots \\
0
\end{array}\right]^{T}+\left[\begin{array}{c}
I \\
-I \\
0 \\
\vdots \\
0
\end{array}\right] S^{T}\left[\begin{array}{c}
F_{2}^{T} \\
F_{3}^{T} \\
0 \\
\vdots \\
0
\end{array}\right]^{T}<0
$$

where $F_{2}$ and $F_{3}$ are auxiliary matrix variables introduced in the proof of Theorem 1 . As seen this method avoids to impose any conservatism to the problem. However, in order to deal with this problem, [16] uses the trivial inequality $-\sigma^{T}(k)(S B)^{-1} \sigma(k)<0$ and replaces this term with zero. Indeed, this would introduce significant conservatism to the sliding function design problem.

The above theorem presents a framework for the design of an ODSMC in order to stabilize the NCS in (1). However, it does not present a bound on the system states. The following theorem aims to provide a bound on the state and the corresponding sliding function of the obtained stochastic closed-loop system.

Theorem 2. In the presence of the exogenous disturbance $d(k)$, if the LMI in (25) is feasible, for the given solution $P>0, Q_{1}>0, Q_{2}>0, L=P^{-1} X_{4}$ and $\rho>0$ of (25), the bound on the augmented system state $\zeta(k)$ is as follows

$$
\forall v>0, \exists k^{\star}>0, \forall k>k^{\star}, \text { s.t. } \mathbb{E}\left\{\|\zeta(k)\|^{2}\right\} \leq \frac{\lambda_{\max }(\mathbf{M})}{\hat{\rho} \lambda_{1}} \gamma+v,
$$


where $\lambda_{1}=\lambda_{\min }\left[\operatorname{diag}\left(P, P, Q_{1}, Q_{2},(S B)^{-1}\right)\right], \mathbf{M}=\operatorname{diag}\left(M_{p}, Q_{1}, Q_{2}\right)$ with $M_{P}=\left[\begin{array}{c}P+\mathbf{R} \\ \mathbf{R}\end{array} \underset{P+\mathbf{R}}{\mathbf{R}}\right]$ and $\mathbf{R}=P B\left(B^{T} P B\right)^{-1} B^{T} P$, and $\gamma=\left(\tau^{2}+\kappa^{2}\right)\left\|\Pi+\Sigma_{c}\right\|\left\|\mathfrak{D}^{b}\right\|^{2}$; here $\Sigma_{c}=\left[\begin{array}{cc}2 B^{T} P B & 0 \\ 0 & 2 \phi B^{T} P B\end{array}\right]$ and the scalar variable $\hat{\rho}>0$ and matrix variable $\Pi=\left[\begin{array}{ll}\Pi_{11} & \Pi_{12} \\ \Pi_{12}^{T} & \Pi_{22}\end{array}\right]>0$ are obtained from solving the following LMI:

$$
\left[\begin{array}{ccccccc}
\Omega_{1} & \star & \star & \star & \star & \star & \star \\
0 & (\hat{\rho}-\rho) I & \star & \star & \star & \star & \star \\
0 & 0 & (\hat{\rho}-\rho) I & \star & \star & \star & \star \\
0 & 0 & 0 & (\hat{\rho}-\rho) I & \star & \star & \star \\
\Sigma_{15}^{T} & \Sigma_{25}^{T} & 0 & \Sigma_{45}^{T} & -\Pi_{11} & \star & \star \\
\Sigma_{16}^{T} & \Sigma_{26}^{T} & 0 & 0 & -\Pi_{12}^{T} & -\Pi_{22} & \star \\
0 & 0 & 0 & 0 & \Omega_{2} & 0 & -\bar{\varepsilon} I
\end{array}\right]<0
$$

where $\bar{\Sigma}_{15}=\lambda P B, \Sigma_{16}=2 \phi A_{\lambda}^{T} P B, \Sigma_{25}=A_{\lambda}^{T} P B+(A-L C)^{T} P B, \Sigma_{26}=-2 \phi A_{\lambda}^{T} P B, \Sigma_{45}=-\theta C^{T} L^{T} P B$, $\Omega_{1}=(\hat{\rho}-\rho) I+\bar{\varepsilon} N^{T} N, \Omega_{2}=2 M^{T} P B, \bar{\varepsilon}>0$ is a scalar variable, and $M$ and $N$ are known matrices in (2).

Proof. Refer to Appendix.

Remark 6. Due to the full column rank of $B$, the columns of $B$ and $P B$ are linearly independent if $P>0$. Therefore, if (12) holds for $P>0$ and $Z$, we have

$$
\operatorname{rank}(Z) \geq \operatorname{rank}(B Z)=\operatorname{rank}(P B) \geq \operatorname{rank}(B)=m,
$$

which implies the non-singularity of $Z$. Furthermore, it can be shown that

$$
Z^{-1}=V \Sigma^{-1} P_{11}^{-1} \Sigma V^{T}
$$

Remark 7. Notice that, in the framework in [16], the cross terms among the system states and the exogenous disturbance have increased the conservatism of the final obtained LMI condition. It has been shown here that the mentioned cross terms should not influence the feasibility region of the final LMI condition (25). Besides, instead of removing the cross terms between $x(k), e(k)$, $x_{c}(k-1)$ and $e_{c}(k-1)$ through several inequalities which were implemented in [16] for $x(k)$ and $x(k-1)$, the cross terms in this paper are kept to be in the original form. This would also widen the feasibility region of the LMI condition presented in this work.

The solution of the LMI in (28) does not have direct influence on the controller design and the actual bound on the system state and/or sliding function. Moreover, if the LMI in (25) is feasible, the LMI in (28) will definitely be feasible and the upper bound presented in (27) exists. Notice also that the solutions of the LMIs (25) can lead us to determine a more accurate bound. Therefore, to obtain the minimum value of the bound in (27), the LMIs in (25) and (28) can be solved subject to a specific criteria. This issue is beyond the scope of this paper and remains for the future work.

Remark 8. The main idea in the proof of Theorems 1 and 2 can be summarized as follows. Firstly, we try to find

$$
\begin{aligned}
& \Delta V(\zeta(k)) \triangleq \mathbb{E}\{V(\zeta(k+1)) \mid \zeta(k)\}-V(\zeta(k)) \\
= & \varpi^{T}(k)\left[\Sigma_{i j}\right]_{6 \times 6} \varpi(k)
\end{aligned}
$$


where $\zeta(k), \varpi(k)$ and $\Sigma_{i j}, i, j=1, \cdots, 6$ are defined in the proof of Theorem 1 . Then, we let

$$
\left[\Sigma_{i j}\right]_{6 \times 6}=\left[\begin{array}{cc}
{\left[\Sigma_{i j}\right]_{4 \times 4}} & \Sigma_{v} \\
\Sigma_{v}^{T} & \Sigma_{c}
\end{array}\right]
$$

where $\Sigma_{c}=\left[\begin{array}{ll}\Sigma_{55} & \Sigma_{56} \\ \Sigma_{56}^{T} & \Sigma_{66}\end{array}\right]$ and $\Sigma_{v}=\left[\begin{array}{cccc}\Sigma_{15}^{T} & \Sigma_{25}^{T} & \Sigma_{35}^{T} & \Sigma_{45}^{T} \\ \Sigma_{16}^{T} & \Sigma_{26}^{T} & \Sigma_{36}^{T} & \Sigma_{46}^{T}\end{array}\right]^{T}$. Now, the switching function matrix $S=B^{T} P$, and the observer gain $L$ can be obtained through solving the LMI condition (25), which is derived by analyzing the feasibility of the following inequality:

$$
\left[\Sigma_{i j}\right]_{4 \times 4}<-\rho I,
$$

where $\rho>0$ is a scalar variable, with the aid of several convexification techniques. The obtained observer-based linear control law in (22) ensures the mean square stability of the augmented closed-loop system (in the absence of the exogenous disturbances). Theorem 2, then, characterizes the boundedness of the augmented closed-loop system when the exogenous disturbances are present, using an innovative scheme. Please also notice that the basic idea in the proof of this theorem comes from Lemma 4.

\section{Numerical examples}

Two numerical examples are presented here in order to evaluate the effectiveness of the proposed ODSMC. All the LMI problems are solved by YALMIP [29] as the interface and SDPT3 [30] as the solver.

\subsection{Example 1}

Consider the system (1) with the following parameters:

$$
\begin{aligned}
& A=\left[\begin{array}{ccc}
0.25 & 0 & 0.28 \\
0 & 1.00 & -0.20 \\
0.50 & 0 & 0.40
\end{array}\right], B=\left[\begin{array}{cc}
1 & 0.2 \\
0.5 & 0 \\
0 & 1
\end{array}\right], C=\left[\begin{array}{ccc}
1 & 0 & 1 \\
0.5 & 1 & 0.3
\end{array}\right], M=\left[\begin{array}{ccc}
0 & 0.1 & -0.1 \\
0 & 0.05 & 0.02 \\
0 & 0 & -0.1
\end{array}\right], \\
& N=\left[\begin{array}{ccc}
0 & 0.15 & 0.01 \\
0.01 & -0.02 & 0 \\
0.01 & 0 & 0.1
\end{array}\right], R(k)=\operatorname{diag}(0.9 \sin (k), 0.6 \sin (k), 0.3 \cos (k)), \bar{\alpha}=0.15, \bar{\beta}=0.2 .
\end{aligned}
$$

Note that the open-loop system is unstable. Suppose

$$
d(k)=\left[\begin{array}{c}
0.01 \\
0.04
\end{array}\right] \sin \left(\frac{k}{10}\right) .
$$

Solving the LMI in (25) gives the following results:

$P=\left[\begin{array}{ccc}32.08 & 0.10 & -1.18 \\ 0.10 & 32.22 & -0.52 \\ -1.18 & -0.52 & 31.48\end{array}\right], Q_{1}=\left[\begin{array}{ccc}17.82 & 3.05 & -2.79 \\ 3.0498 & 3.30 & 1.98 \\ -2.79 & 1.98 & 17.70\end{array}\right], Q_{2}=\left[\begin{array}{ccc}10.64 & 3.05 & -1.00 \\ 3.05 & 2.35 & 1.07 \\ -1.00 & 1.07 & 13.16\end{array}\right]$,

$S=\left[\begin{array}{ccc}32.13 & 16.21 & -1.44 \\ 5.23 & -0.50 & 31.24\end{array}\right], L=\left[\begin{array}{cc}0.15 & -0.00 \\ -0.13 & 0.27 \\ 0.18 & 0.05\end{array}\right], \rho=0.16, \varepsilon=23.60$ 

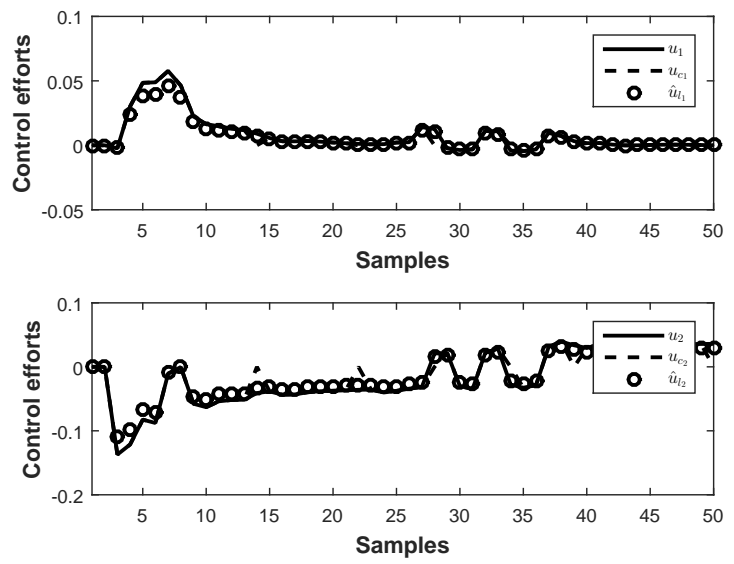

Fig. 2. Control effort in Example 1
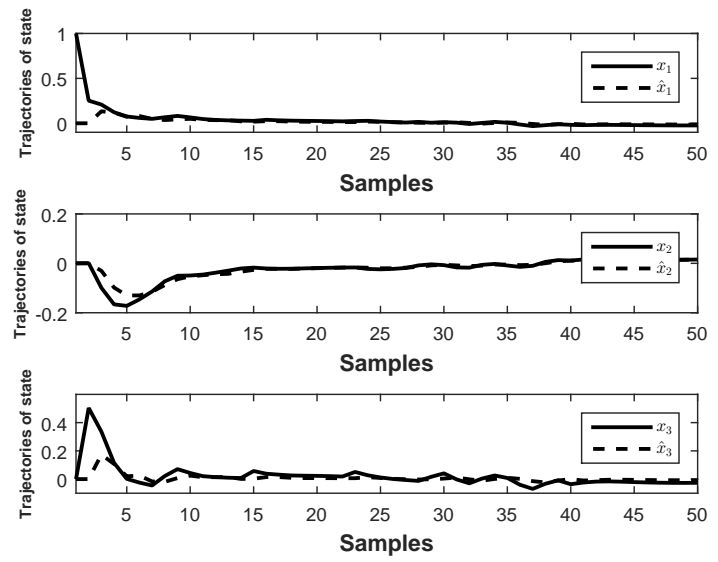

Fig. 3. Trajectories of the system state and state estimate in Example 1

The component $\vartheta(k)$ in (18) is assumed to be as

$$
\vartheta(k)=\mathfrak{D}^{a}+\eta \operatorname{diag}\left(\mathfrak{D}^{b}\right) \operatorname{sgn}(S \hat{x}(k)),
$$

where $\operatorname{diag}\left(\mathfrak{D}^{b}\right)=\operatorname{diag}\left(d_{1}^{b}, \cdots, d_{m}^{b}\right)$ and $\eta>0$ is a scalar. Hence, using $P, \mathfrak{D}^{a}=\left[\begin{array}{ll}0 & 0\end{array}\right]^{T}, \mathfrak{D}^{b}=$ $\left[\begin{array}{ll}0.01 & 0.04\end{array}\right]^{T}, \lambda=0.3$ and $\eta=0.1$ the control law given in (18) and (32) is obtained. The results of applying this controller to the system (1) are shown in Figs. 2-4. Here, the initial state is assumed to be $x(0)=\left[\begin{array}{lll}1 & 0 & 0\end{array}\right]^{T}$. Bernoulli sequences $\alpha(k)$ and $\beta(k)$ are depicted in Fig. 5. It can be seen that the proposed ODSMC law successfully drives the state trajectories toward the ideal sliding surface and keeps them in a boundary layer around the sliding surface thereafter.

Moreover, solving the LMI in (28) for $\Pi$ and $\hat{\rho}$ as a feasibility problem in Matlab, with the obtained solutions of the LMI (25), and further, letting $\tau=1.1, \kappa=0.1$ in (20) and (21), the ultimate bound on $\mathbb{E}\left\{\|\zeta(k)\|^{2}\right\}$ in (27) is equal to $6.92 \times 10^{6}$. However, it is worth mentioning that, as we stated before, to obtain the minimum value of the bound in (27), the LMIs in (25) and (28) should be solved subject to a specific criteria. This is not an easy deal and is beyond the scope of this paper. 

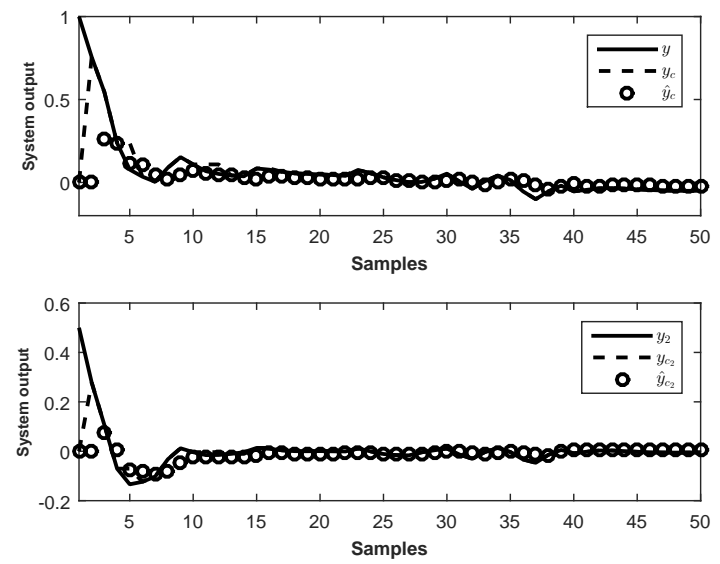

Fig. 4. Trajectories of the system output in Example 1
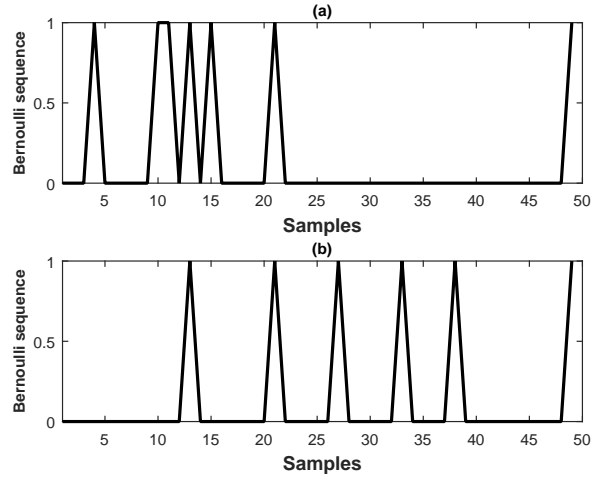

Fig. 5. Bernoulli sequences a) $\alpha(k), b) \beta(k)$ in Example 1 

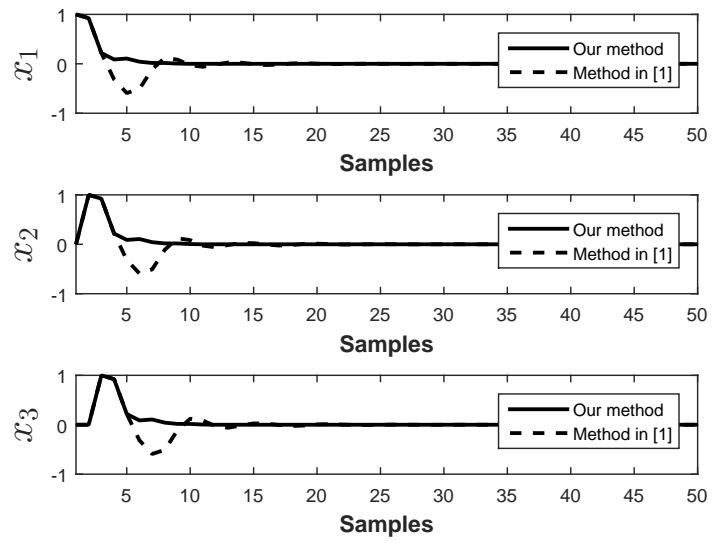

Fig. 6. Trajectories of the system state in Example 2

\subsection{Example 2}

We consider the control problem of uninterruptible power system (UPS) in [1]. The objective is to govern the pulse width-modulated inverter, so that the output AC voltage of UPS remains constant at a desired set-point with minimum distortion. The considered UPS has the capacity of $1 \mathrm{kVA}$. The discrete-time model is obtained by using the sampling time $10 \mathrm{~ms}$ at half-load operating point as:

$$
A=\left[\begin{array}{ccc}
0.9226 & -0.6330 & 0 \\
1.0 & 0 & 0 \\
0 & 1.0 & 0
\end{array}\right], B=\left[\begin{array}{l}
1 \\
0 \\
0
\end{array}\right], C=\left[\begin{array}{lll}
23.738 & 20.287 & 0
\end{array}\right]
$$

As in this paper the exogenous disturbance is assumed to be the matched one, we set $B=B_{1}=$ $B_{2}=\left[\begin{array}{lll}1 & 0 & 0\end{array}\right]^{T}$. Also $d(k)=\frac{1}{k^{2}}$. Notice also that as the system in [1] does not include unmatched uncertainty we also set $\Delta A=0$ in this example. In addition, we temporarily assume that the state observer and the controller are not located in the same place; see Remark 2. Besides, in order for a fair comparison with the control scheme in [1], which is applicable to small random delays, both the occurrence probabilities of the random measurement packet losses and the actuation packet losses are assumed as $\bar{\alpha}=0.1, \bar{\beta}=0.1$. The initial conditions are $x(0)=\left[\begin{array}{lll}1 & 0 & 0\end{array}\right]^{T}$ and $\hat{x}(0)=$ $\left[\begin{array}{lll}0 & 0 & 0\end{array}\right]^{T}$. In our method we set $\lambda=0.3$ and $\vartheta(k)=0$. The simulation results of the state responses are given in Fig. 6 and the Bernoulli sequences $\alpha(k)$ and $\beta(k)$ are shown in Fig. 7. As seen in Fig. 6, the ODSMC developed in this paper leads to a better closed-loop transient response compared to the $\mathscr{H}_{\infty}$ state feedback proposed in [1]. Notice also that, since $\tau=0$ and $\kappa=0$ in (20) and (21), the ultimate bound in (27) will be zero.

\section{Conclusions}

This paper proposes a robust observer-based discrete-time sliding mode control by utilizing only output signals for the networked systems involving random consecutive packet losses in both the measurement and actuation channels. We have exploited Bernoulli random binary distribution to model the consecutive data packet dropouts. Besides, the proposed method, achieved with the aid 

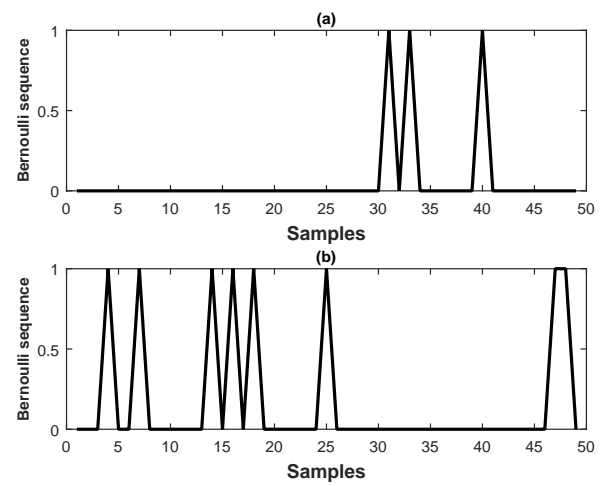

Fig. 7. Bernoulli sequences a) $\alpha(k), b) \beta(k)$ in Example 2

of an LMI scheme, forms a unified framework for the robust ODSMC design. Furthermore, it has reduced the conservatism of the existing methods in the literature. For analyzing the ultimate boundedness of the derived closed-loop system, the notion of exponential mean square stability has been utilized. Numerical examples have been presented to show the effectiveness of the proposed scheme.

\section{References}

1 Yang, F. W., Wang, Z. D., Hung, Y. S., and Gani, M.: "H $H_{\infty}$ control for networked systems with random communication delays", IEEE Trans. Autom. Control, 2006, 51, (3), pp. 511-518.

2 Goodwin, G. C., Haimovich, H., Quevedo, D. E., and Welsh, J. S.: “A moving horizon approach to networked control system design", IEEE Trans. Autom. Control, 2004, 49, (9), 1427-1445.

3 Nilsson, J., Bernhardsson, B., and Wittenmark, B.: "Stochastic analysis and control of realtime systems with random time delays", Automatica, 1998, 34, (1), pp. 57-64.

4 Luan, X., Shi, P., and Liu, F.: "Stabilization of networked control systems with random delays", IEEE Trans. Ind. Elec., 2011, 58, (9), pp. 4323-4330.

5 Walsh, G. C., Ye, H., and Bushnell, L. G.: "Stability analysis of networked control systems", IEEE Trans. Control Syst. Technol., 2002, 10, (3), pp. 438-446.

6 Wang, Z. and Yang, F.: "Robust filtering for uncertain linear systems with delayed states and outputs", IEEE Trans. Circuits Syst. I, 2002, 49, (1), pp. 125-130.

7 Kim, S. H. and Parkg, P.: "Networked-based robust $\mathrm{H}_{\infty}$ control design using multiple levels of network traffic", Automatica, 2009, 45, (3), pp. 764-770.

8 Tsai, N. and Ray, A.: "Stochastic optimal control under randomly varying delays", Int. J. Control, 1997, 69, (5), pp. 1179-1202.

9 Ray, A.: "Output feedback control under randomly varying distributed delays", J. Guid. Control Dyna., 1994, 17, (4), pp. 701-711.

10 Koshkouei, A. and Zinober, A. S. I.: "Sliding mode control of discrete-time systems", Journal of Dynamic Systems, Measurement, and Control, 2000, 122, pp. 793-802. 
11 Sapturk, S. Z., Istefanopulous, Y., and Kaynak, O.: "On the stability of discrete-time sliding mode control systems", IEEE Transactions on Automatic Control, 1987, 32, pp. 930-932.

12 Gao, W., Wang, Y., and Homaifa, A.: "Discrete-time variable structure control system", IEEE Trans. Ind. Electron, 1995, 42, pp. 117-122.

13 Hui, S. and Zak, S. H.: "On discrete-time variable structure sliding mode control”, Systems and Control Letters, 1999, 38, pp. 283-288.

14 Spurgeon, S. K.: "Hyperplane design techniques for discrete-time variable structure control systems", International Journal of Control, 1992, 55(2), pp. 445-456.

15 Monsees, G.: Discrete-time sliding mode control (Ph.D. Thesis, Delft University of Technolog, 2002, 1st edn.).

16 Niu, Y. and Ho, D. W. C.: "Design of Sliding Mode Control Subject to Packet Losses", IEEE Transactions on Automatic Control, 2010, 55, pp. 2623-2628.

17 Chen, B., Niu, Y., and Zou, Y.: "Sliding mode control for networked systems with Markovian jumping parameters". Proc. Proc.12th International Conference on Control, Automation, Robotics and Vision, Guangzhou, China, Dec. 2012, pp. 1495-1500.

18 Niu, Y., Ho, D. W. C, and Lam, J.: "Robust integral sliding mode control for uncertain stochastic systems with time-varying delay", Automatica, 2005, 41, pp. 873-880.

19 Bartoszewicz, A.: "Discrete-time quasi-sliding-mode control strategies", IEEE Transactions on Industrial Electronics, 1998, 45, pp. 633-637.

20 Govindaswamy, S., Spurgeon, S. K., and Floquet, T.: "Discrete-time output feedback slidingmode control design for uncertain systems using linear matrix inequalities", International Journal of Control, 2011, 84, 916-930.

21 Schenato, L., Sinopoli, B., Franceschetti, M., Poolla, K., and Sastry, S. S.: "Foundations of control and estimation over lossy networks", Proc. IEEE, Jan. 2007, 95, (1), 163-187.

22 Sinopoli, B., Schenato, L., Franceschetti, M., Poolla, K., Jordan, M. I., and Sastry, S. S.: "Kalman filtering with intermittent observations", IEEE Trans. Autom. Control, Sep. 2009, 49, (9), 1453-1463.

23 Schenato, L.: "To zero or to hold control inputs with lossy links?", IEEE Trans. Autom. Control, May 2009, 54, (5), 1093-1099.

24 Peterson, I. R.: "A stabilization algorithm for a class of uncertain linear systems", Systems Control Lett., 1987, 8, 351-357.

25 Li, L., Ugrinovskii, V. A., and Orsi, R.: "Decentralized robust control of uncertain Markov jump parameter systems via output feedback", Automatica, 2007, 43, pp. 1932-1944.

26 Ho, D. W. C. and Lu, G.: "Robust stabilization for a class of discrete-time nonlinear systems via output feedback: The unified LMI approach", Int. J. Control, 2003, 76, pp. 105-115.

27 Lai, N. O., Edwards, C., and Spurgeon, S. K.: "Discrete output feedback sliding-mode control with integral action", Int. J. Robust Nonlinear Control, 2006, 16, pp. 21-43.

28 Edwards, C.: "A practical method for the design of sliding mode controllers using linear matrix inequalities", Automatica, 2004, 40, pp. 1761-1769.

29 Löfberg, J.: "YALMIP: A toolbox for modeling and optimization in MATLAB". Proc. CCA/ISIC/CACSD, September 2004, URL: http://control . ee.ethz . ch/index . cgi?action=details; id= 2088 ; page=publications. 
30 Toh, K. C., Todd, M., Ttnc, R., and Tutuncu, R. H.: "SDPT3 - a MATLAB software package for semidefinite programming", Optimization Methods and Software, 1998, 11, pp. 545-581.

\section{Appendices}

\subsection{Proof of Theorem 1}

Define

$$
\begin{aligned}
V(\zeta(k))= & x^{T}(k) P x(k)+e^{T}(k) P e(k)+x_{c}^{T}(k-1) Q_{1} x_{c}(k-1) \\
& +e_{c}^{T}(k-1) Q_{2} e_{c}(k-1)+\sigma^{T}(k)(S B)^{-1} \sigma(k),
\end{aligned}
$$

where $\zeta(k)=\left[x^{T}(k) e^{T}(k) x_{c}^{T}(k-1) e_{c}^{T}(k-1) \sigma^{T}(k)\right]^{T}, P>0, Q_{1}>0$ and $Q_{2}>0$ are symmetric matrices and $S=B^{T} P$. Thus, it can be written

$$
\begin{aligned}
& \Delta V(\zeta(k)) \triangleq \mathbb{E}\{V(\zeta(k+1)) \mid \zeta(k)\}-V(\zeta(k)) \\
= & \mathbb{E}\left\{x^{T}(k+1) P x(k+1)+e^{T}(k+1) \operatorname{Pe}(k+1)+x_{c}^{T}(k) Q_{1} x_{c}(k)+e_{c}^{T}(k) Q_{2} e_{c}(k)\right. \\
& \left.+\sigma^{T}(k+1)(S B)^{-1} \sigma(k+1) \mid \zeta(k)\right\}-x^{T}(k) \operatorname{Px}(k)-e^{T}(k) \operatorname{Pe}(k)-x_{c}^{T}(k-1) Q_{1} x_{c}(k-1) \\
& -e_{c}^{T}(k-1) Q_{2} e_{c}(k-1)-\sigma^{T}(k)(S B)^{-1} \sigma(k) .
\end{aligned}
$$

It is then followed by

$$
\begin{aligned}
& \mathbb{E}\left\{x^{T}(k+1) P x(k+1) \mid \zeta(k)\right\} \\
= & \mathbb{E}\left\{x^{T}(k)\left[A+\Delta A-\hat{A}_{\Delta}+\Delta A_{\lambda}+\frac{\beta-\bar{\beta}}{1-\bar{\beta}} \hat{A}\right]^{T} P\left[A+\Delta A-\hat{A}_{\Delta}+\Delta A_{\lambda}+\frac{\beta-\bar{\beta}}{1-\bar{\beta}} \hat{A}\right] x(k) \mid \zeta(k)\right\} \\
& +2 x^{T}(k)\left[\left(\lambda I_{n}+\Delta A\right)^{T} S^{T}(S B)^{-1} S A_{\lambda}-\phi A_{\lambda}^{T} S^{T}(S B)^{-1} S A_{\lambda}\right] e(k)+2 x^{T}(k)\left(\Delta A+\lambda I_{n}\right)^{T} S^{T} d_{\vartheta}(k) \\
& +2 \phi x^{T}(k) A_{\lambda}^{T} S^{T} \vartheta(k)+2 e^{T}(k) A_{\lambda}^{T} S^{T} d_{\vartheta}(k)-2 \phi e^{T}(k) A_{\lambda}^{T} S^{T} \vartheta(k)+(1+\phi) e^{T}(k) A_{\lambda}^{T} S^{T}(S B)^{-1} S \\
& \times A_{\lambda} e(k)+d_{\vartheta}(k)^{T}(S B) d_{\vartheta}(k)+\phi \vartheta \\
= & x^{T}(k)[(A)(S B) \vartheta(k) \\
& \left.+\phi A_{\lambda}^{T} S^{T}(S B)^{-1} S A_{\lambda}\right] x(k)+2 x^{T}(k)\left[\left(\lambda I_{n}+\Delta A\right)^{T} S^{T}(S B)^{-1} S A_{\lambda}-\phi A_{\lambda}^{T} S^{T}(S B)^{-1} S A_{\lambda}\right] e(k) \\
& +2 x^{T}(k)\left(\Delta A+\lambda I_{n}\right)^{T} S^{T} d_{\vartheta}(k)+2 \phi x^{T}(k) A_{\lambda}^{T} S^{T} \vartheta(k)+2 e^{T}(k) A_{\lambda}^{T} S^{T} d_{\vartheta}(k)-2 \phi e^{T}(k) A_{\lambda}^{T} S^{T} \vartheta(k) \\
& +(1+\phi) e^{T}(k) A_{\lambda}^{T} S^{T}(S B)^{-1} S A_{\lambda} e(k)+d_{\vartheta}(k)^{T}(S B) d_{\vartheta}(k)+\phi \vartheta^{T}(k)(S B) \vartheta(k)
\end{aligned}
$$


where $\hat{A}_{\Delta}=B(S B)^{-1} S(A+\Delta A), \Delta A_{\lambda}=B(S B)^{-1} S\left(\lambda I_{n}+\Delta A\right)$ and $\phi=\frac{\bar{\beta}}{1-\bar{\beta}}$. Besides, note that $\mathbb{E}\{(\beta-\bar{\beta})\}=0$ and $\mathbb{E}\left\{(\beta-\bar{\beta})^{2}\right\}=\bar{\beta}(1-\bar{\beta}) \triangleq \delta^{2}$. Thus, it can be demonstrated that

$$
\begin{aligned}
& \mathbb{E}\left\{e^{T}(k+1) P e(k+1) \mid \zeta(k)\right\} \\
= & x^{T}(k)\left[\Delta A^{T} P \Delta A+\phi A_{\lambda}^{T} P B\left(B^{T} P B\right)^{-1} B^{T} P A_{\lambda}+\theta(L C)^{T} P L C\right] x(k) \\
& +e^{T}(k)\left[(A-L C)^{T} P(A-L C)+\phi A_{\lambda}^{T} P B\left(B^{T} P B\right)^{-1} B^{T} P A_{\lambda}\right] e(k)+\theta x_{c}^{T}(k-1)(L C)^{T} P L C x_{c}(k-1) \\
& +\theta^{2} e_{c}^{T}(k-1)(L C)^{T} P L C e_{c}(k-1)+d_{\vartheta}^{T}(k) B^{T} P B d_{\vartheta}(k)+\phi \vartheta^{T}(k) B^{T} P B \vartheta(k) \\
& +2 x^{T}(k)\left[\Delta A^{T} P(A-L C)-\phi A_{\lambda}^{T} P B\left(B^{T} P B\right)^{-1} B^{T} P A_{\lambda}\right] e(k)-2 \theta x^{T}(k)(L C)^{T} P L C x_{c}(k-1) \\
& -2 \theta x^{T}(k) \Delta A^{T} P L C e_{c}(k-1)+2 x^{T}(k) \Delta A^{T} P B d_{\vartheta}(k)+2 \phi x^{T}(k) A_{\lambda}^{T} P B \vartheta(k) \\
& -2 \theta e^{T}(k)(A-L C)^{T} P L C e_{c}(k-1)+2 e^{T}(k)(A-L C)^{T} P B d_{\vartheta}(k)-2 \phi e^{T}(k) A_{\lambda}^{T} P B \vartheta(k) \\
& -2 \theta e_{c}^{T}(k-1) C^{T} L^{T} P B d_{\vartheta}(k),
\end{aligned}
$$

in which again $\mathbb{E}\{(\alpha-\bar{\alpha})\}=0$ and $\mathbb{E}\left\{(\alpha-\bar{\alpha})^{2}\right\}=\bar{\alpha}(1-\bar{\alpha}) \triangleq \psi^{2}$. Also

$$
\begin{aligned}
& \mathbb{E}\left\{x_{c}^{T}(k) Q_{1} x_{c}(k) \mid \zeta(k)\right\} \\
= & (1-\bar{\alpha}) x^{T}(k) Q_{1} x(k)+\bar{\alpha} x_{c}^{T}(k-1) Q_{1} x_{c}(k-1),
\end{aligned}
$$

and

$$
\begin{aligned}
& \mathbb{E}\left\{e_{c}^{T}(k) Q_{2} e_{c}(k) \mid \zeta(k)\right\} \\
= & \mathbb{E}\left\{\left[-\alpha x(k)+\alpha x_{c}(k-1)+e(k)\right]^{T} Q_{2}\left[-\alpha x(k)+\alpha x_{c}(k-1)+e(k)\right] \mid \zeta(k)\right\} \\
= & \bar{\alpha} x^{T}(k) Q_{2} x(k)-2 \bar{\alpha} x^{T}(k) Q_{2} x_{c}(k-1)-2 \bar{\alpha} x^{T}(k) Q_{2} e(k)+\bar{\alpha} x_{c}^{T}(k-1) Q_{2} x_{c}(k-1) \\
& +2 \bar{\alpha} x_{c}^{T}(k-1) Q_{2} e(k)+e^{T}(k) Q_{2} e(k),
\end{aligned}
$$

in which $\mathbb{E}\left\{\alpha^{2}\right\}=\bar{\alpha}$. Besides, we have

$$
\begin{aligned}
& \mathbb{E}\left\{\sigma^{T}(k+1)(S B)^{-1} \sigma(k+1) \mid \zeta(k)\right\} \\
= & x^{T}(k)\left[\theta C^{T} L^{T} S^{T}(S B)^{-1} S L C+\lambda^{2} S^{T}(S B)^{-1} S\right] x(k)+e^{T}(k)(\lambda I-L C)^{T} S^{T}(S B)^{-1} S(\lambda I-L C) e(k) \\
& +\theta x_{c}^{T}(k-1) C^{T} L^{T} S^{T}(S B)^{-1} S L C x_{c}(k-1)+\theta^{2} e_{c}^{T}(k-1) C^{T} L^{T} S^{T}(S B)^{-1} S L C e_{c}(k-1) \\
& -2 x^{T}(k) \lambda S^{T}(S B)^{-1} S(\lambda I-L C) e(k)-2 \theta x^{T}(k) C^{T} L^{T} S^{T}(S B)^{-1} S L C x_{c}(k-1) \\
& +2 \theta \lambda x^{T}(k) S^{T}(S B)^{-1} S L C e_{c}(k-1)-2 \theta e^{T}(k)(\lambda I-L C)^{T} S^{T}(S B)^{-1} S L C e_{c}(k-1) .
\end{aligned}
$$

It is also easy to show that

$$
\begin{aligned}
& \sigma^{T}(k)(S B)^{-1} \sigma(k)=[x(k)-e(k)]^{T} S^{T}(S B)^{-1} S[x(k)-e(k)] \\
& =x^{T}(k) S^{T}(S B)^{-1} S x(k)-2 x^{T}(k) S^{T}(S B)^{-1} S e(k)+e^{T}(k) S^{T}(S B)^{-1} S e(k) .
\end{aligned}
$$

Using (35)-(40) and defining $\varpi(k)=\left[\begin{array}{llllll}x^{T}(k) & e^{T}(k) & x_{c}^{T}(k-1) & e_{c}^{T}(k-1) & d_{\vartheta}^{T}(k) & \vartheta^{T}(k)\end{array}\right]^{T}$, (34) can be rearranged as

$$
\Delta V(\zeta(k))=\varpi^{T}(k)\left[\Sigma_{i j}\right]_{6 \times 6} \varpi(k),
$$


where

$$
\begin{aligned}
\Sigma_{11}= & (A+\Delta A)^{T} P(A+\Delta A)-(A+\Delta A)^{T} S^{T}(S B)^{-1} S(A+\Delta A)+\left(\lambda I_{n}+\Delta A\right)^{T} S^{T}(S B)^{-1} S\left(\lambda I_{n}+\Delta A\right) \\
& +\Delta A^{T} P \Delta A+2 \phi A_{\lambda}^{T} P B\left(B^{T} P B\right)^{-1} B^{T} P A_{\lambda}-\left(1-\lambda^{2}\right) S^{T}(S B)^{-1} S+\theta(L C)^{T} P L C \\
& +\theta C^{T} L^{T} S^{T}(S B)^{-1} S L C-P+(1-\bar{\alpha}) Q_{1}+\bar{\alpha} Q_{2}, \\
\Sigma_{12}= & \left(\lambda I_{n}+\Delta A\right)^{T} S^{T}(S B)^{-1} S A_{\lambda}+\Delta A^{T} P(A-L C)+S^{T}(S B)^{-1} S-2 \phi A_{\lambda}^{T} P B\left(B^{T} P B\right)^{-1} B^{T} P A_{\lambda} \\
& -\bar{\alpha} Q_{2}-\lambda S^{T}(S B)^{-1} S(\lambda I-L C), \\
\Sigma_{13}= & -\theta(L C)^{T} P L C-\theta C^{T} L^{T} S^{T}(S B)^{-1} S L C-\bar{\alpha} Q_{2}, \\
\Sigma_{14}= & -\theta \Delta A^{T} P L C+\theta \lambda S^{T}(S B)^{-1} S L C, \\
\Sigma_{15}= & \left(\Delta A+\lambda I_{n}\right)^{T} S^{T}+\Delta A^{T} P B, \\
\Sigma_{16}= & 2 \phi A_{\lambda}^{T} P B, \\
\Sigma_{22}= & (1+2 \phi) A_{\lambda}^{T} S^{T}(S B)^{-1} S A_{\lambda}+(\lambda I-L C)^{T} S^{T}(S B)^{-1} S(\lambda I-L C)+(A-L C)^{T} P(A-L C) \\
& -S^{T}(S B)^{-1} S-P+Q_{2}, \\
\Sigma_{23}= & \bar{\alpha} Q_{2}, \\
\Sigma_{24}= & -\theta(A-L C)^{T} P L C-\theta(\lambda I-L C)^{T} S^{T}(S B)^{-1} S L C, \\
\Sigma_{25}= & A_{\lambda}^{T} S^{T}+(A-L C)^{T} P B, \\
\Sigma_{26}= & -2 \phi A_{\lambda}^{T} P B, \\
\Sigma_{33}= & \theta(L C)^{T} P L C+\theta C^{T} L^{T} S^{T}(S B)^{-1} S L C+\bar{\alpha} Q_{2}-(1-\bar{\alpha}) Q_{1}, \\
\Sigma_{34}= & 0, \\
\Sigma_{35}= & 0, \\
\Sigma_{36}= & 0, \\
\Sigma_{44}= & \theta^{2}(L C)^{T} P L C+\theta^{2} C^{T} L^{T} S^{T}(S B)^{-1} S L C-Q_{2}, \\
&
\end{aligned}
$$

and $\Sigma_{45}=-\theta C^{T} L^{T} P B, \Sigma_{46}=0, \Sigma_{55}=2 S B, \Sigma_{56}=0, \Sigma_{66}=2 \phi S B$. Now, to prove the system stability, let $\left[\begin{array}{c}d_{\vartheta}(k) \\ \vartheta(k)\end{array}\right]=0$. Then the system is stable if

$$
\Xi:=\left[\Sigma_{i j}\right]_{4 \times 4}<-\rho I
$$

where $\rho>0$ is a scalar variable. With the choice of $S=B^{T} P$ and utilizing the Schur complement, it can be shown that the feasibility of (42) is equivalent to that of

$$
\left[\begin{array}{cccccccccc}
\bar{\Sigma}_{11} & \star & \star & \star & \star & \star & \star & \star & \star & \star \\
\bar{\Sigma}_{12}^{T} & \bar{\Sigma}_{22} & \star & \star & \star & \star & \star & \star & \star & \star \\
-\bar{\alpha} Q_{2} & \bar{\alpha}_{2} & \bar{\Sigma}_{33} & \star & \star & \star & \star & \star & \star & \star \\
0 & 0 & 0 & -Q_{2}+\rho I & \star & \star & \star & \star & \star & \star \\
B^{T} P\left(\lambda I_{n}+\Delta A\right) & B^{T} P A_{\lambda} & 0 & 0 & -B^{T} P B & \star & \star & \star & \star & \star \\
-\lambda B^{T} P & B^{T} P(\lambda I-L C) & 0 & -\theta B^{T} P L C & 0 & -B^{T} P B & \star & \star & \star & \star \\
-2 \phi B^{T} P A_{\lambda} & 2 \phi B^{T} P A_{\lambda} & 0 & 0 & 0 & 0 & -2 \phi B^{T} P B & \star & \star & \star \\
P \Delta A & P(A-L C) & 0 & -\theta P L C & 0 & 0 & 0 & -P & \star & \star \\
\theta P L C & 0 & -\theta P L C & 0 & 0 & 0 & 0 & 0 & -\theta P & \star \\
\theta B^{T} P L C & 0 & -\theta B^{T} P L C & 0 & 0 & 0 & 0 & 0 & 0 & -\theta B^{T} P B
\end{array}\right]<0
$$


where $\theta=\frac{\bar{\alpha}}{1-\bar{\alpha}}, \phi=\frac{\bar{\beta}}{1-\bar{\beta}}$, and

$$
\begin{aligned}
\bar{\Sigma}_{11}= & (A+\Delta A)^{T} P(A+\Delta A)-(A+\Delta A)^{T} S^{T}(S B)^{-1} S(A+\Delta A)-S^{T}(S B)^{-1} S-P \\
& +\bar{\alpha} Q_{2}+(1-\bar{\alpha}) Q_{1}+\rho I, \\
\bar{\Sigma}_{12}= & S^{T}(S B)^{-1} S-\bar{\alpha} Q_{2} \\
\bar{\Sigma}_{22}= & -S^{T}(S B)^{-1} S-P+Q_{2}+\rho I, \\
\bar{\Sigma}_{33}= & -(1-\bar{\alpha}) Q_{1}+\bar{\alpha} Q_{2}+\rho I .
\end{aligned}
$$

Hence, using Lemma 3 it can be shown that the feasibility of the inequality in (43) is equivalent to that of

$$
\left[\begin{array}{ccc}
\hat{\Sigma}_{11} & \hat{\Sigma}_{12} & \cdots \\
\hat{\Sigma}_{12}^{T} & \hat{\Sigma}_{22} & \cdots \\
\vdots & \vdots & \ddots
\end{array}\right]<0
$$

with

$$
\begin{aligned}
\hat{\Sigma}_{11}= & \left(A+\Delta A+B F_{1}\right)^{T} P\left(A+\Delta A+B F_{1}\right)-P+(1-\bar{\alpha}) Q_{1}+F_{2}^{T}\left(B^{T} P B\right) F_{2}+F_{2}^{T} B^{T} P+P B F_{2} \\
& +\bar{\alpha} Q_{2}+\rho I, \\
\hat{\Sigma}_{12}= & F_{2}^{T} B^{T} P B F_{3}-F_{2}^{T} B^{T} P+P B F_{3}-\bar{\alpha} Q_{2} \\
\hat{\Sigma}_{22}= & F_{3}^{T}\left(B^{T} P B\right) F_{3}-F_{3}^{T} B^{T} P-P B F_{3}-P+Q_{2}+\rho I,
\end{aligned}
$$

where $F_{1}, F_{2}$ and $F_{3}$ are auxiliary variables [25] and note that except $\hat{\Sigma}_{11}, \hat{\Sigma}_{12}$ and $\hat{\Sigma}_{22}$ other entries of (44) are the same as their counterparts in (43). Thus, using Lemma 5, (45) can be rearranged as

$$
\begin{aligned}
\hat{\Sigma}_{11}= & {\left[P(A+\Delta A)+B Z F_{1}\right]^{T} P^{-1}\left[P(A+\Delta A)+B Z F_{1}\right]-P+(1-\bar{\alpha}) Q_{1}+F_{2}^{T} Z^{T} B^{T} P^{-1} B Z F_{2} } \\
& +F_{2}^{T} Z^{T} B^{T}+B Z F_{2}+\bar{\alpha} Q_{2}+\rho I, \\
\hat{\Sigma}_{12}= & F_{2}^{T} Z^{T} B^{T} P^{-1} B Z F_{3}-F_{2}^{T} Z^{T} B^{T}+B Z F_{3}-\bar{\alpha} Q_{2}, \\
\hat{\Sigma}_{22}= & F_{3}^{T} Z^{T} B^{T} P^{-1} B Z F_{3}-F_{3}^{T} Z^{T} B^{T}-B Z F_{3}-P+Q_{2}+\rho I,
\end{aligned}
$$

where $Z$ satisfies $P B=B Z$. Using the Schur complement it can be seen that (44) is implied by the following inequality,
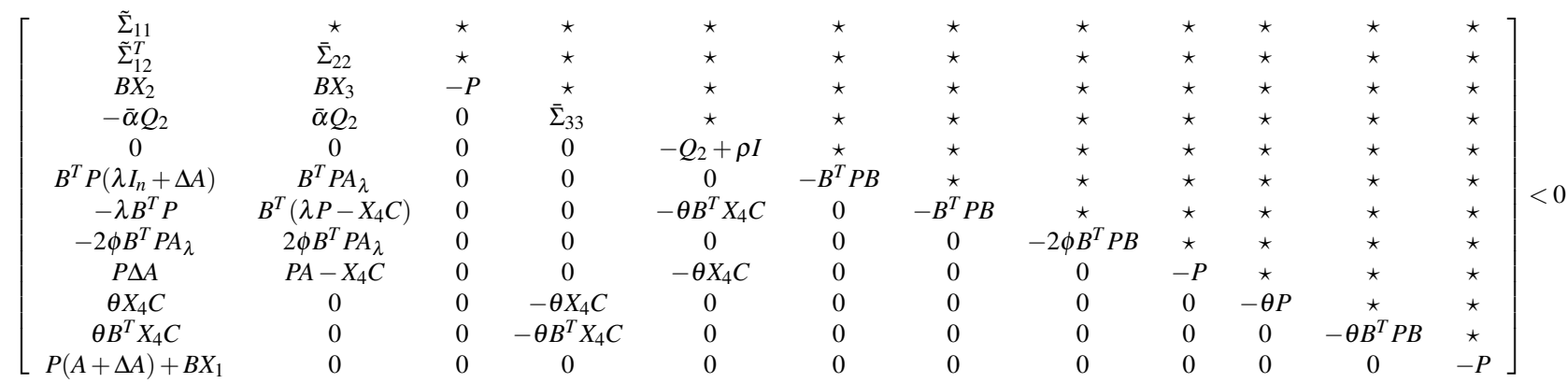

where $\tilde{\Sigma}_{11}=-P+(1-\bar{\alpha}) Q_{1}+\bar{\alpha} Q_{2}+X_{2}^{T} B^{T}+B X_{2}+\rho I, \tilde{\Sigma}_{12}=-X_{2}^{T} B^{T}+B X_{3}-\bar{\alpha} Q_{2}, \tilde{\Sigma}_{22}=$ $-X_{3}^{T} B^{T}-B X_{3}-P+Q_{2}+\rho I, X_{1}=Z F_{1}, X_{2}=Z F_{2}, X_{3}=Z F_{3}$ and $X_{4}=P L$. With the help of Lemma 1 and assuming $\Delta A_{k}$ satisfies the condition in (2), (47) is also sufficed by the LMI in (25). 


\subsection{Proof of Theorem 2}

Defining $v(k)=\left[\begin{array}{llll}x^{T}(k) & e^{T}(k) & x_{c}^{T}(k-1) & e_{c}^{T}(k-1)\end{array}\right]^{T}$ and $\Sigma_{v}=\left[\begin{array}{cccc}\Sigma_{15}^{T} & \Sigma_{25}^{T} & \Sigma_{35}^{T} & \Sigma_{45}^{T} \\ \Sigma_{16}^{T} & \Sigma_{26}^{T} & \Sigma_{36}^{T} & \Sigma_{46}^{T}\end{array}\right]^{T}$ (see the proof of Theorem 1), according to Lemma 2 , we have

$$
2 v^{T}(k) \Sigma_{v}\left[\begin{array}{c}
d_{\vartheta}(k) \\
\vartheta(k)
\end{array}\right] \leq v^{T}(k) \Sigma_{v} \Pi^{-1} \Sigma_{v}^{T} v(k)+\left[\begin{array}{c}
d_{\vartheta}(k) \\
\vartheta(k)
\end{array}\right]^{T} \Pi\left[\begin{array}{c}
d_{\vartheta}(k) \\
\vartheta(k)
\end{array}\right],
$$

where $\Pi>0$ with appropriate dimension. It follows from (41), (42) and (48) that

$$
\Delta V(\zeta(k)) \leq-v^{T}(k)\left[\rho I-\Sigma_{v} \Pi^{-1} \Sigma_{v}^{T}\right] v(k)+\left[\begin{array}{c}
d_{\vartheta}(k) \\
\vartheta(k)
\end{array}\right]^{T}\left[\Sigma_{c}+\Pi\right]\left[\begin{array}{c}
d_{\vartheta}(k) \\
\vartheta(k)
\end{array}\right](k),
$$

in which $\Sigma_{c}=\left[\begin{array}{cc}\Sigma_{55} & \Sigma_{56} \\ \Sigma_{56}^{T} & \Sigma_{66}\end{array}\right]$. If we choose $\Pi>0$ such that

$$
\hat{\rho} I<\rho I-\Sigma_{v} \Pi^{-1} \Sigma_{v}^{T},
$$

where $\rho>\hat{\rho}>0$, which is always possible if $\rho>0$ exists, then, it follows from (49) that

$$
\Delta V(\zeta(k)) \leq-\hat{\rho} v^{T}(k) v(k)+\left[\begin{array}{c}
d_{\vartheta}(k) \\
\vartheta(k)
\end{array}\right]^{T}\left[\Sigma_{c}+\Pi\right]\left[\begin{array}{c}
d_{\vartheta}(k) \\
\vartheta(k)
\end{array}\right]
$$

Moreover, note that as $\sigma(k)=S(x(k)-e(k))$ we can derive

$$
V(\zeta(k))=v^{T}(k) \mathbf{M} v(k),
$$

where $\mathbf{M}=\operatorname{diag}\left(M_{p}, Q_{1}, Q_{2}\right)$ with $M_{P}=\left[\begin{array}{cc}P+\mathbf{R} & \mathbf{R} \\ \mathbf{R} & P+\mathbf{R}\end{array}\right]$ and $\mathbf{R}=P B\left(B^{T} P B\right)^{-1} B^{T} P$, hence,

$$
\lambda_{\min }(\mathbf{M})\|v(k)\|^{2} \leq V(\zeta(k)) \leq \lambda_{\max }(\mathbf{M})\|v(k)\|^{2} .
$$

Additionally, it is known that

$$
\lambda_{1}\|\zeta(k)\|^{2} \leq V(\zeta(k)) \leq \lambda_{2}\|\zeta(k)\|^{2} .
$$

where $\lambda_{1}=\lambda_{\min }\left(\operatorname{diag}\left(P, P, Q_{1}, Q_{2},(S B)^{-1}\right)\right)$ and $\lambda_{2}=\lambda_{\max }\left(\operatorname{diag}\left(P, P, Q_{1}, Q_{2},(S B)^{-1}\right)\right)$. Hence, from (51) and (53) one can derive that

$$
\Delta V(\zeta(k)) \leq-\frac{\hat{\rho}}{\lambda_{\max }(\mathbf{M})} V(\zeta(k))+\gamma
$$

where $\gamma=\left(\tau^{2}+\kappa^{2}\right)\left\|\Pi+\Sigma_{c}\right\|\left\|\mathfrak{D}^{b}\right\|^{2}$. Note that from (42) it can simply be written that $\forall v(k) \neq 0$

$$
\begin{aligned}
\boldsymbol{v}^{T}(k) \Xi \boldsymbol{v}(k) & =\mathbb{E}\left\{V(\zeta(k+1)) \mid \begin{array}{c}
d_{\vartheta}(k) \\
\vartheta(k)
\end{array}\right]=0 \\
& <-\rho(k)\}-V(\zeta(k)) \\
& <v^{T}(k) \boldsymbol{v}(k) .
\end{aligned}
$$

It is known that $\mathbb{E}\left\{\left.V(\zeta(k+1))\right|_{\left[\begin{array}{c}d_{\vartheta}(k) \\ \vartheta(k)\end{array}\right]=0} \mid \zeta(k)\right\}>0$, and thus, from (56) and (53), it can be claimed that $\rho<\lambda_{\max }(\mathbf{M})$. Hence,

$$
\frac{\hat{\rho}}{\lambda_{\max }(\mathbf{M})}<1 .
$$


Finally, from Lemma 4 and (55), we can find the bound given in (27).

Furthermore, to find $\Pi>0$ in (50), defining $\Pi=\left[\begin{array}{ll}\Pi_{11} & \Pi_{12} \\ \Pi_{12}^{T} & \Pi_{22}\end{array}\right]$, we can rewrite this inequality, for given $P>0, Q_{1}>0, Q_{2}>0, L$ and $\rho>0$, as

$$
\left[\begin{array}{cccccc}
(\hat{\rho}-\rho) I & \star & \star & \star & \star & \star \\
0 & (\hat{\rho}-\rho) I & \star & \star & \star & \star \\
0 & 0 & (\hat{\rho}-\rho) I & \star & \star & \star \\
0 & 0 & 0 & (\hat{\rho}-\rho) I & \star & \star \\
\Sigma_{15}^{T} & \Sigma_{25}^{T} & 0 & \Sigma_{45}^{T} & -\Pi_{11} & \star \\
\Sigma_{16}^{T} & \Sigma_{26}^{T} & 0 & 0 & -\Pi_{12}^{T} & -\Pi_{22}
\end{array}\right]<0 .
$$

Using Lemma 1, (57) can be sufficed by the LMI (28). 